\title{
Appendix.
}

\section{Proof of Proposition}

We introduce some notations. Let $d N_{t}$ denote the arrival of paper at $t$ (i.e., $d N_{t}=1\left(d N_{t}=0\right)$ represents that a paper arrives (does not arrive) at $t$ ). Let $V(k, t)$ denote the value function of the editor who already accepted $k$ papers, i.e., $V(k, t)$ is the maximum expected quality of remaining $(K-k)$ papers under the editor's optimal policy. For boundary conditions, it is straightforward to show that

$$
V(K, t)=0 \text { for all } t \text { and } V(k, T)=0 \text { for all } k \text {. }
$$

Noting that after the editor accepts $(k+1)$-th paper at $t$, the value function shifts from $V(k, t)$ to $V(k+1, t)$, and the difference should be compensated by the threshold:

$$
\underline{q}(k, t)=V(k, t)-V(k+1, t) .
$$

We can restrict that $q$ is distributed smoothly over $[0, \infty) .{ }^{1}$ Hence, we have the boundary condition of $\underline{q}(K-1, T)=0$, which in conjunction with (1) and (2) yields that

$$
\underline{q}(k, T)=0 \text { for all } k .
$$

It is convenient to use $g(\underline{q})$, defined as follows:

$$
g(\underline{q})=\mathbb{E}[(q-\underline{q}) \mathbf{1}(q>\underline{q})] .
$$

Note the the distribution of $q$ is smooth, $g(\underline{q})$ has the following property:

$$
g(\underline{q})>0 \text { and } \frac{\partial g(\underline{q})}{\partial \underline{q}}<0
$$

Next, we examine the dynamics of $q(k, t)$. The value function satisfies the following:

$$
V(k, t)=\mathbb{E}\left[(q+V(k+1, t+d t)) \mathbf{1}(q>\underline{q}(k, t)) d N_{t}+V(k, t+d t)\left(1-\mathbf{1}(q>\underline{q}(k, t)) d N_{t}\right)\right],
$$

\footnotetext{
${ }^{1}$ Since the editor wants to maximize the overall quality of accepted papers, the editor can treat papers with negative qualities as non-arrival of papers.
} 
which in conjunction with (4) yields that

$$
\frac{\partial V(k, t)}{\partial t}=-\lambda g(\underline{q}(k, t))
$$

From (2), we have

$$
\frac{\partial V(k, t)}{\partial t}=\frac{\partial \underline{q}(k, t)}{\partial t}+\frac{\partial V(k+1, t)}{\partial t}
$$

which along with (6) implies that

$$
\begin{aligned}
\frac{\partial \underline{q}(k, t)}{\partial t} & =-\lambda g(\underline{q}(k, t))+\lambda g(\underline{q}(k+1, t)) \text { for } k<K-1 \\
\frac{\partial \underline{q}(K-1, t)}{\partial t} & =-\lambda g(\underline{q}(K-1, t)) \leq 0
\end{aligned}
$$

Now, we are ready to show $\underline{q}(k, t) \leq \underline{q}(k+1, t)$. We use mathematical induction. First, the followings are satisfied when $k+1=K-1$ :

$$
\begin{aligned}
\underline{q}(k+1, T) & =0 \\
\frac{\partial \underline{q}(k+1, t)}{\partial t} & \leq 0 \\
\underline{q}(k+1, T)-\underline{q}(k, T) & =0,
\end{aligned}
$$

where the first and last conditions are from (3) and the second is from (8).

Second, we show that under the conditions of (9)-(11), it holds that $\underline{q}(k, t) \leq \underline{q}(k+1, t)$. Define $\Delta_{k}(s)=q(k+1, T-s)-q(k, T-s)$ for $s \in[0, T]$. Note that $\Delta_{k}(0) \geq 0$ from (11). Fix $s_{0} \geq 0$. Assume that $\Delta_{n}\left(s_{0}\right) \geq 0$. Then, it holds that

$$
\begin{aligned}
\left.\frac{\partial \Delta_{k}}{\partial s}\right|_{s=s_{0}} & =-\frac{\partial \underline{q}(k+1, T-s)}{\partial(T-s)}+\frac{\partial \underline{q}(k, T-s)}{\partial(T-s)} \geq\left.\frac{\partial q(k, T-s)}{\partial(T-s)}\right|_{s=s_{0}} \\
& =-\lambda g\left(q\left(k, T-s_{0}\right)\right)+\lambda g\left(q\left(k+1, T-s_{0}\right)\right) \\
& =-\lambda g\left(q\left(k+1, T-s_{0}\right)-\Delta_{k}\left(s_{0}\right)\right)+\lambda g\left(q\left(k+1, T-s_{0}\right)\right)
\end{aligned}
$$

where the first inequality is from (10), the second equality is from (8) and the last equality is from 
the definition of $\Delta_{k}(s)$. The above inequality shows that when $\Delta_{k}(s) \downarrow 0$, the lower bound of $\frac{\partial \Delta_{k}}{\partial s}$ approaches to zero, preventing $\Delta_{k}(s)$ from going below zero. Hence, $\Delta_{k}(s) \geq 0$ for $s \in[0, T]$, implying that

$$
\underline{q}(k, t) \leq \underline{q}(k+1, t) .
$$

Lastly, we complete the induction by confirming the following:

$$
\begin{aligned}
\underline{q}(k, T) & =0 \\
\frac{\partial \underline{q}(k, t)}{\partial t} & \leq 0 \\
\underline{q}(k, T)-\underline{q}(k-1, T) & =0,
\end{aligned}
$$

where the first and last conditions are from (3). The second inequality is obtained by applying (5) and (12) into (7). Hence, from mathmatical induction, it holds that $\underline{q}(k, t) \leq \underline{q}(k+1, t)$ for all $k$. This completes the proof of the proposition. 